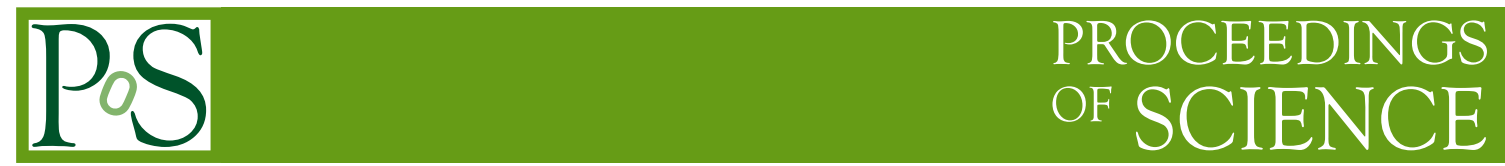

\title{
Direct photons at ATLAS
}

\section{Francesca BUCCI, on behalf of the ATLAS Collaboration*}

University of Geneva, Geneva

E-mail: f.bucci@cern.ch

Direct photon studies provide an ideal arena for testing predictions of perturbative QCD and to constrain the gluon parton density function, particularly at large $\mathrm{x}$. In addition, direct photon processes represent an important contribution in estimating the background to other physics processes and provide important calibration methods for hadronic jets. The motivation for direct photon studies at ATLAS will be discussed together with photon identification techniques.

2008 Physics at LHC

September 29 - 4 October 2008

Split, Croatia

${ }^{*}$ Speaker. 


\section{ATLAS: A Toroidal LHC ApparatuS}

ATLAS is a general purpose detector at the Large Hadron Collider (LHC) at CERN. The LHC is a $27 \mathrm{~km}$ ring colliding bunches of up to $10^{11}$ protons at a rate of $40 \mathrm{MHz}$. It is designed to provide $\sqrt{s}=14 \mathrm{TeV}$ collisions at a luminosity of $10^{34} \mathrm{~cm}^{-2} \mathrm{~s}^{-1}$. The ATLAS detector consists of an inner tracker surrounded by a $2 \mathrm{~T}$ solenoid magnet, a Lead-Liquid Argon electromagnetic calorimeter (ECAL), a scintillating tile hadronic calorimeter and a muon spectrometer system [1].

The ECAL is the most important subdetector in the study of direct photon production. It extends up to $|\eta|<3.2$ and it is made up of three different layers: the first consists of $\Delta \eta \times \Delta \phi=$ $0.003 \times 0.1$ strips, the second of $\Delta \eta \times \Delta \phi=0.025 \times 0.025$ square cells which contain most of the energy deposited by an electromagnetic object, and the third of coarser $0.05 \times 0.025$ cells used to estimate the leakage into the hadronic calorimeter.

\section{Direct photon theory and motivation}

Direct photons are defined as photons produced in the primary parton-parton interaction. At the leading order (LO) they originate from quark-gluon Compton scattering and quark-antiquark annihilation (figure 1). The next to leading order (NLO) process primarily comes from photon radiation from a quark line. The event signature consists of a well isolated photon and at least one jet opposite in the azimuthal direction. The main background comes from di-jet events in which one jet fragments into light neutral mesons (mostly $\pi^{0}$ and $\eta$ ) decaying into two photons. We will refer to these jets as "fake" photons.
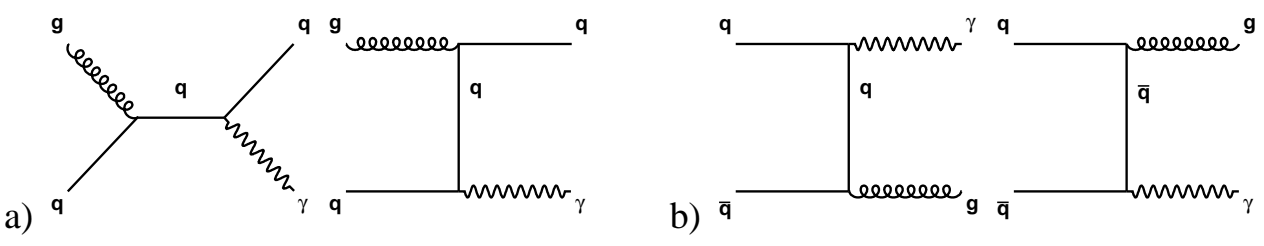

Figure 1: Leading order Feynman diagrams of the a) Compton b) Annihilation processes

Direct photons find application both in physics studies and detector calibration. Physics studies include searches for new physics, where deviation from theoretical predictions could bear evidence of new processes like SUSY, $H \rightarrow \gamma \gamma$ or improve sensitivity to triple gauge coupling [2]. For detector calibration the energy balance between the photon and the recoiling hadronic energy could be used to tune the jet energy scale corrections [1].

An important motivation is probing the gluon parton density function (PDF) as the direct photon production cross section depends on the gluon momentum distribution in the proton. At LHC this will be possible in a wide kinematic region extending in the range $\sim 10^{2}<Q^{2}<10^{8} \mathrm{GeV}^{2}$. As shown in previous analyses [3] the direct photon cross section has an $\eta$ dependence which is sensitive to the various parameterizations of the gluon PDF. The CTEQ6 PDF [4] is the latest generation of parton distributions from the CTEQ collaboration. It contains the standard sets and the eigenvector sets that characterize the uncertainties. A Monte Carlo study of the scattering kinematics of direct photons shows variations of $4 \%-10 \%$ in the photon $\eta$ distribution in the $p_{\mathrm{T}}$ and $\eta$ ranges accessible by ATLAS when comparing the various parameterizations of the gluon PDF [5]. 


\section{Photon identification}

Direct photon analysis makes use of a cut-based identification method to separate real photons from fake photons. Photons appear as narrow objects in the ECAL while fake photons have a broader profile and can deposit a substantial fraction of their energy in the hadronic calorimeter. Hence, cuts applied on longitudinal and transverse shower shape variables and on hadronic leakage can be used to reject fake photons. In particular, information from the fine strips in the ECAL help to identify substructures in the showers to efficiently distinguish between photons from the hard scatter and photons from $\pi^{0}$ decays. Each cut is optimized in $p_{\mathrm{T}}$ and $\eta$ bins. The overall efficiency reached using the cut-based method is $\sim 80-90 \%$ (figure 2 a).

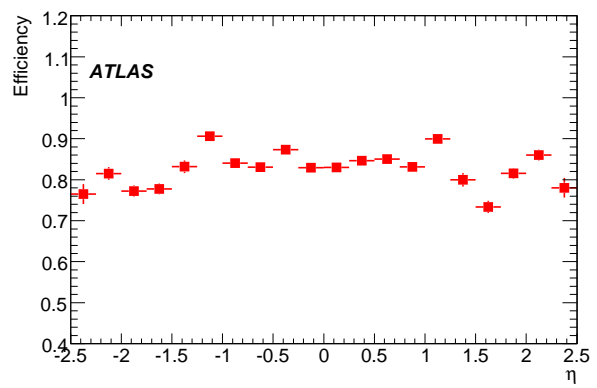

a)

Figure 2: a) Efficiency as a function of the pseudorapidity for photons with $E_{\mathrm{T}}>25 \mathrm{GeV}$ from $H \rightarrow \gamma \gamma$; b) $E_{\mathrm{T}}$ distribution of fake photon candidates in jets after different cuts [2].

Figure 2 b) shows the level of background after the hadronic leakage cut (Had) and the cuts on the shower shape variables constructed using the second (S2) and first (S1) layer of the ECAL. The main contribution comes from $\pi^{0}$ that account for $\sim 70 \%$ of the remaining fake photons after all cuts. To further reduce this contribution an explicit isolation cut can be applied. For example requiring sum of all tracks with $p_{\mathrm{T}}>1 \mathrm{GeV}$ within a cone of 0.3 around the cluster centroid to be less than $4 \mathrm{GeV}$ gives an additional rejection factor of 1.5 to 2 for a relatively small efficiency loss [2].

\section{References}

[1] The ATLAS Collaboration, G. Aad et al., The ATLAS Experiment at the CERN Large Hadron Collider, JINST 3 (2008) S08003

[2] The ATLAS Collaboration, Expected Performance of the ATLAS Experiment, Detector, Trigger and Physics, CERN-OPEN-2008-020, Geneva (December 2008), arXiv:0901.0512v1 [hep-ex]

[3] A. Kumar et al., Study of direct photon production at the CERN LHC, Physical Review D, Volume 67, (2003) 014016

[4] J. Pumplin et al., JHEP 0207, 012 (2002)

[5] T. I. Hollins, SCT hybrid testing and the production of direct photons in the ATLAS experiment at the LHC, CERN-THESIS-2007-061 